\title{
Editorial
}

\section{Two Hundred Treasured Issues!}

JAD is no longer a startup-having completed 200 issues over 17 years and now appearing semimonthly-leading us to consider how we have done in implementing JAD's original vision. In the editorial by Merril and Ghanbari [1], the beginning of JAD is recollected as a venue for the most original ideas in Alzheimer's disease (AD), something that did not exist in the 1990s. Together with Mark Smith, we set a broad net to encourage the most innovative researchers in AD to join JAD's family and be editors in recruiting the best new ideas. Number of articles, impact factor, citations, and famous authors were all secondary to ingenuity and to publishing work that advances our field. We think JAD's success inspired the numerous journals now in our field to create a super highway to get new ideas to the forefront. As JAD continues to develop, it is critical that advancement in AD research continues.

In the coming months, JAD will be introducing groundbreaking changes in editorial structure and tools that will advance the field. We welcome your thoughts and thank you for the sustained support.

George Perry

\section{REFERENCE}

[1] Merril CR, Ghanbari HA (2015) Two hundred issues later: The dream comes true. J Alzheimers Dis 44, 727-728. 